Sharif University of Technology
Scientia Iranica
Transactions E: Industrial Engineering
http://scientiairanica.sharif.edu

\title{
Impact of measurement error on mixed EWMA- CUSUM control chart
}

\author{
M. Noor-ul-Amin* \\ Department of Statistic, COMSATS University Islamabad, Lahore Campus. \\ Received 7 May 2019; received in revised form 1 July 2020; accepted 26 October 2020
}

\section{KEYWORDS \\ CUSUM; \\ EWMA control chart; \\ Measurement error; \\ Monte-Carlo \\ simulation method; \\ Average run length.}

\begin{abstract}
In statistical process control, measurement error plays a key role that is usually ignored. Measurement error can yield incorrect conclusions about the performance of the process. This study examined the effect of measurement error on the shift detection ability of the mixed Exponentially Weighted Moving Average-Cumulative Sum (EWMA-CUSUM) control chart. Then, it investigated the performance of the mixed EWMA-CUSUM chart in case of mean shift through (i) covariate method, (ii) multiple measurement method, and (iii) linearly increasing variance method. The performance measurement tools such as Average Run Length (ARL) and Standard Deviation of Run Length (SDRL) were estimated using the Monte-Carlo simulation method. It was concluded that the performance of the mixed EWMA-CUSUM control chart was adversely affected by considering the measurement error. It was revealed from the comparative study that the mixed EWMA-CUSUM control chart outperformed the EWMA and CUSUM control charts in the presence of measurement error. An illustrative example was presented to demonstrate the performance of control charts in case of measurement error.
\end{abstract}

(C) 2022 Sharif University of Technology. All rights reserved.

\section{Introduction}

The objective of every manufacturing industry is to attain process stability by reducing the variation in the production process. The causes of variation in process output are divided into two categories: (i) common cause and (ii) special cause. The process is said to be out-of-control when an assignable cause is present. Statistical Process Control (SPC) tools are used to detect an assignable cause or special cause of variation in the process outcome. Control charts are the statistical tools used in manufacturing processes to detect an out-of-control situation so that the correc-

\footnotetext{
*.E-mail address: nooramin.stats@gmail.com
}

doi: $10.24200 /$ sci.2020.53453.3244 tive measures may be taken in time. Exponentially Weighted Moving Average (EWMA) and Cumulative Sum (CUSUM) control charts use pervious information of samples along with current sample information to monitor the process parameters. This property makes them more sensitive than the Shewhart control chart to detect small to moderate shifts. Roberts [1] was the first who introduced the EWMA control chart. Page [2] was the first who studied the performance of the CUSUM control chart. There are a variety of literature-based findings on the EWMA and CUSUM charts for efficient monitoring of process parameters to enhance the detecting ability of the control charts. Researchers have suggested various control charts; for instance, Abbas et al. [3] presented a mixed EWMACUSUM chart for the process mean. Abujiya et al. [4] proposed a mixed Shewhart-EWMA chart for location. 
Zaman et al. [5] proposed a mixed CUSUM-EWMA control chart for process location. Ajadi et al. [6] further increased the sensitivity of mixed EWMACUSUM control chart for location parameter. Riaz et al. [7] extended the mixed EWMA-CUSUM control chart using an auxiliary variable.

The control charts are used to observe the changes in the production process due to assignable causes and the variation due to measurement error which is usually ignored. The variation due to measurement error can adversely affect the performance of the control charts, as mentioned by Linna and Woodall [8] as well as Maravelakis et al. [9]. The variation due to measurement error needs considerable attention. Mittag and Stemann [10] studied the effect of measurement error on a joint $\bar{X}-S$ control chart by assuming the model $Y=X+\varepsilon$, where $X$ is the actual value of the variable of interest and $Y$ is the measured value because of the random error $\varepsilon$. Linna and Woodall [8] explored the idea of measurement error with covariates and linearly increasing variance strategy on Shewhart control charts using a previous model, i.e., $Y=X+\varepsilon$, where $\varepsilon$ is a random error and $Y$ is the study variable. Maravelakis et al. [9] investigated the performance of the EWMA control chart in the presence of measurement error, assuming a model with covariates and taking multiple measurements and they proved that in case of measurement error, the performance of the chart in terms of monitoring the mean was adversely affected. Maravelakis [11] studied the effect of measurement error on CUSUM control chart and according to his findings, CUSUM was highly affected by measurement error. The author concluded that multiple measurements reduced the effect of measurement error on the chart performance, while in the presence of linearly increasing variance, the CUSUM chart performance improved. Recently, Noor-ul-Amin et al. [12] studied the effect of measurement error on the auxiliary information-based EWMA control chart.

In this paper, the effect of measurement error on the performance of a mixed EWMA-CUSUM control chart was examined for monitoring the process mean using the covariate method, multiple measurements, and linearly increasing variance method. Section 2 presents the basic concepts of classical EWMA, CUSUM, and mixed EWMA-CUSUM control charts. Section 3 gives a description of measurement error with EWMA, CUSUM, and mixed EWMA-CUSUM control charts using (i) covariates method, (ii) multiple measurements, and (iii) linearly increasing variance method. Section 4 explores the effect of measurement error on a mixed EWMA-CUSUM control chart using different sample sizes, i.e. $(n=1,3,5)$, at $A R L_{0}=500$. Section 5 presents the application of EWMA, CUSUM, and mixed EWMA-CUSUM control charts to a real dataset in the presence of measurement error. Section 6 discusses the comparative results of EWMA, CUSUM, and mixed EWMA-CUSUM control charts.

\section{Classical control charts}

This section presents the introduction of classical EWMA, CUSUM, and mixed EWMA-CUSUM control charts for monitoring the process mean.

\subsection{EWMA control chart}

The statistic for the EWMA control chart is defined as follows:

$$
z_{i}=\lambda \bar{y}_{i}+(1-\lambda) z_{i-1}, \quad z_{0}=\mu
$$

where $i=1,2,3, \ldots$ and $\bar{y}_{i}$ is the mean of the observations taken from the sample and $\lambda$ is a smoothing parameter that lies between 0 and 1 . Here, $\mu$ and $\sigma$ are the population mean and standard deviation, respectively, and $z_{0}$ is the initial value, which is taken equal to $\mu$ or average of Phase-I data in case $\mu_{0}$ is unknown. The control limits for EWMA statistic are as follows:

$$
\begin{aligned}
& U C L=\mu+L \frac{\sigma}{\sqrt{n}} \sqrt{\frac{\lambda}{2-\lambda}\left(1-(1-\lambda)^{2 i}\right)}, \\
& L C L=\mu-L \frac{\sigma}{\sqrt{n}} \sqrt{\frac{\lambda}{2-\lambda}\left(1-(1-\lambda)^{2 i}\right)},
\end{aligned}
$$

where $L$ is the control constant used to specify the width of limits when the process is in-control; the mean of the EWMA statistic is $\mu$; and the standard deviation is given as follows:

$$
\frac{\sigma}{\sqrt{n}} \sqrt{\frac{\lambda}{2-\lambda}\left(1-(1-\lambda)^{2 i}\right)} .
$$

For large values of $i$, the limits in Eq. (2) and Eq. (3) converge to the constant limits such that:

$$
\begin{aligned}
& U C L=\mu+L \frac{\sigma}{\sqrt{n}} \sqrt{\frac{\lambda}{2-\lambda}}, \\
& L C L=\mu-L \frac{\sigma}{\sqrt{n}} \sqrt{\frac{\lambda}{2-\lambda}} .
\end{aligned}
$$

\subsection{CUSUM control chart}

CUSUM control chart used V-mask and tabular form procedures to monitor the process location. This notion was originally introduced by Page [2]. The statistics for the CUSUM control chart are given as:

$$
\begin{aligned}
C_{i}^{+} & =\max \left[0,\left(\bar{y}_{i}-\mu\right)-K+C_{i-1}^{+}\right], \\
C_{i}^{-} & =\max \left[0,-\left(\bar{y}_{i}-\mu\right)-K+C_{i-1}^{-}\right],
\end{aligned}
$$

where $C_{i}^{+}$and $C_{i}^{-}$are the upper and lower statistics, respectively, and $K$ is the reference value for the 
CUSUM chart, often taken equal to half of the shift detected (cf., Ewan and Kemp [13]). The starting value for both plotting statistics is taken zero and $C_{i}^{-}$ and $C_{i}^{+}$are plotted against the control limit $H$; if $C_{i}^{+}>H$ or $C_{i}^{-}>H$ for any value of $i$, the process is considered to be out-of-control. The two parameters $K$ and $H$ are defined in a standardized manner (cf., Montgomery [14]):

$$
K=k \sigma, \quad H=h \sigma,
$$

where $k$ and $h$ are two constants selected carefully because the CUSUM chart is very sensitive to these constants.

\subsection{Mixed EWMA-CUSUM control chart}

Abbas et al. [3] presented a mixed EWMA-CUSUM control chart for process monitoring. In this scheme, the statistic for the location is defined as follows:

$$
\begin{aligned}
& M_{i}^{+}=\max \left[0,\left(Q_{i}-\mu\right)-K_{q}^{\prime}+M_{i+1}^{+}\right], \\
& M_{i}^{-}=\max \left[0,\left(Q_{i}-\mu\right)-K_{q}^{\prime}+M_{i+1}^{-}\right],
\end{aligned}
$$

where $M_{i}^{-}$and $M_{i}^{+}$are the lower and upper statistics for the mixed EWMA-CUSUM chart and initially set to zero. The $Q_{i}$ is the EWMA statistic that is given by:

$$
\begin{aligned}
& Q_{i}=\lambda \bar{Y}_{i}+(1-\lambda) Q_{i-1}, \quad Q_{0}=0, \\
& E\left(Q_{i}\right)=\mu_{0}, \quad \sigma^{2}\left(Q_{i}\right)=\frac{\sigma^{2}}{n}\left[\frac{\lambda}{2-\lambda}\left(1-(1-\lambda)^{2 i}\right)\right],
\end{aligned}
$$

where $M_{i}^{+}$and $M_{i}^{-}$are plotted against the control limit $H_{q}^{\prime}$. If $M_{i}^{+}$is plotted above $H_{q}^{\prime}$, the process mean is said to be shifted above the target mean; however, should be plotted above $H_{q}^{\prime}$, the process mean is said to be shifted below the target mean:

$$
K_{q}^{\prime}=k_{q} \sqrt{\operatorname{var}\left(Q_{i}\right)}, \quad H_{q}^{\prime}=h_{q} \sqrt{\operatorname{var}\left(Q_{i}\right)} .
$$

\section{Measurement error using covariate model}

Bennett [15] studied the performance of the Shewhart chart for the mean using the measurement error model given in Eq. (11) such that:

$$
Y=X+\varepsilon \text {, }
$$

where $X$ is the true value of the quality characteristic assumed to be normal with mean $\mu$ and variance $\sigma^{2}$ when the process is in-control and $\varepsilon$ is a random error due to measurement imprecision. It is assumed that $Y$ and $X$ are distributed with the same mean but different variances and the variance of $Y$ is greater than that of $X$. However, we are not able to observe this true value but rather a value of $Y$ which is related to $X$. The linear model with covariate is defined as follows:

$$
Y=A+B X+\varepsilon,
$$

where $A$ and $B$ are two constants and $\varepsilon$ is a random error that is normally distributed with mean zero and variance $\sigma_{m}^{2}$. Eq. (11) is the special case of this covariate model with $A=0$ and $B=1$, and it is assumed that all parameters are known (cf., Linna and Woodall [8]).

\subsection{EWMA control chart with covariate}

Linna and Woodall [8] assumed that $Y$ and $X$ are linearly correlated, i.e., $Y=A+B X+\varepsilon$, where $\varepsilon$ is the random error and independent of $X$ and $Y$ is normally distributed with mean $A+B \mu$ and variance $B^{2} \sigma^{2}+\sigma_{m}^{2}$ while the assumption is that $A, B, \sigma^{2}$ and $\sigma_{m}^{2}$ are known. For the construction of the control chart, we can keep the variable $X$ under control for measured quantity $Y$. Maravelakis et al. [9] introduced the statistic for the EWMA control chart with covariate as given below:

$$
z_{i}=\lambda \bar{Y}_{i}+(1-\lambda) z_{i-1}, \quad z_{0}=A+B \mu_{0}
$$

where $z_{0}$ is the initial value taken equal to the $A+$ $B \mu_{0}$ or average of initial data when $\mu_{0}$ is unknown. The control limits for the EWMA control chart with covariate are given below:

$$
\begin{aligned}
U C L= & A+B \mu \\
& +L \sqrt{\frac{\lambda}{2-\lambda}\left[1-(1-\lambda)^{2 i}\right] \frac{B^{2} \sigma^{2}+\sigma_{m}^{2}}{n}}, \\
L C L= & A+B \mu \\
& -L \sqrt{\frac{\lambda}{2-\lambda}\left[1-(1-\lambda)^{2 i}\right] \frac{B^{2} \sigma^{2}+\sigma_{m}^{2}}{n}},
\end{aligned}
$$

for large values of $i$, these limits in Eqs. (14) and (15) converge to constant limits given below:

$$
\begin{aligned}
& U C L=A+B \mu+L \sqrt{\frac{\lambda}{2-\lambda} \frac{B^{2} \sigma^{2}+\sigma_{m}^{2}}{n}}, \\
& L C L=A+B \mu-L \sqrt{\frac{\lambda}{2-\lambda} \frac{B^{2} \sigma^{2}+\sigma_{m}^{2}}{n}} .
\end{aligned}
$$

\subsection{CUSUM control chart with covariate}

Maravelakis [11] defined the upper-sided CUSUM control chart for detecting an increase in the process mean:

$$
S_{i}^{+}=\max \left(0, S_{i-1}^{+}+\bar{y}_{i}-k_{z}^{+}\right),
$$

and the corresponding lower-sided CUSUM chart for detecting a decrease in the process mean:

$$
S_{i}^{-}=\min \left(0, S_{i-1}^{-}+\bar{y}_{i}+k_{z}^{-}\right) \text {, }
$$

where $S_{0}^{+}=S_{0}^{-}=0$ and the value of $k_{z}^{+}$is given by Hawkins and Olwell [16]:

$$
k_{z}^{+}=A+B \frac{\mu+\mu_{1}}{2}, \quad k_{z}^{+}=k_{z}^{-},
$$

where $\mu_{1}$ denotes the out-of-control mean and represents anticipated mean shift. The critical value is denoted by $h_{z}$. It is assumed that $h_{z}^{+}=h_{z}^{-}$. 


\subsection{Multiple measurements}

There are certain conditions under which multiple measurements are taken per sampling unit rather than taking only a single measurement per item. This approach is recommended by Walden [17] as a strategy to overcome the effect of measurement error. By using multiple measurements, chances for success are high in terms of statistical power and precision (cf., Linna and Woodall [8]).

\subsubsection{EWMA control chart with multiple measurements}

Maravelakis et al. [9] studied the effect of multiple measurements on EWMA control chart. The EWMA statistic with multiple measurements is given by:

$$
Q_{i}^{+}=\lambda \overline{\bar{Y}}_{i}+(1-\lambda) Q_{i-1}^{+}, \quad Q_{0}=A+B \mu
$$

where $\overline{\bar{Y}}_{i}$ is the mean of the observations collected at time $i$. Linna and Woodall [8] proved the variance of the overall mean for multiple measurements as follows:

$$
\frac{B^{2} \sigma^{2}}{n}+\frac{\sigma_{m}^{2}}{n p},
$$

and the limiting form of the control limits for EWMA control chart with multiple measurements is given below:

$$
\begin{aligned}
& U C L=A+B \mu+L \sqrt{\frac{\lambda}{2-\lambda}\left(\frac{B^{2} \sigma^{2}}{n}+\frac{\sigma_{m}^{2}}{n p}\right)}, \\
& L C L=A+B \mu-L \sqrt{\frac{\lambda}{2-\lambda}\left(\frac{B^{2} \sigma^{2}}{n}+\frac{\sigma_{m}^{2}}{n p}\right)} .
\end{aligned}
$$

\subsubsection{CUSUM control chart with multiple measurements}

Maravelakis [11] studied the effect of multiple measurements on CUSUM control chart. It is assumed that by using the model in Eq. (12) with mean zero and variance $\sigma_{m}^{2}$, the variable $\bar{Y}_{i}$ has mean $A+B \mu$ and variance $\frac{B^{2} \sigma^{2}+\sigma_{m}^{2}}{n}$. The statistics for CUSUM with multiple measurements are given in Eqs. (18) and (19).

\subsection{Linear increasing variance method}

In the covariates model, it is assumed that the variance is constant, which is not working in all industrial problems because the variance of the measurement process depends on the mean level of the process in some situations. Montgomery and Runger [18] and Linna and Woodall [8] pointed to a situation where this phenomenon might occur in industrial problems. If the variance of variable $Y$ changes linearly with the variable $X$ then it is assumed that $\varepsilon$ is distributed normally with mean 0 and variance $C+D \mu$, where $C$ and $D$ are two known constants. The variable $y_{i}$ is normally distributed with mean $A+B \mu$ and variance $\left(B^{2} \sigma^{2}+C+D \mu\right)$. The $D$ parameter has a key role in changing the process mean underlying measurements (cf., Maravelakis et al. [9]).

Maravelakis et al. [9] used the same statistic given in Eq. (21) for the linearly increasing variance method and presented the control limits for EWMA statistic with the linearly increasing variance method as follows:

$$
\begin{aligned}
& U C L=A+B \mu+L \sqrt{\frac{\lambda}{2-\lambda}\left(\frac{B^{2} \sigma+C+D \mu}{n}\right)}, \\
& L C L=A+B \mu-L \sqrt{\frac{\lambda}{2-\lambda}\left(\frac{B^{2} \sigma+C+D \mu}{n}\right)} .
\end{aligned}
$$

Maravelakis [11] discussed the CUSUM control chart by using the model Eq. (12) with the same assumptions for linearly increasing variance with mean zero and variance $C+D \mu$, where $C$ and $D$ are constant and to be known. Then, $Y$ is normally distributed with mean $A+B \mu$ and variance $B^{2} \sigma^{2}+C+D \mu$; the statistics are the same as those defined in Eqs. (18) and (19).

\section{Proposed control chart}

This section presents the mixed EWMA-CUSUM control chart in the presence of measurement error. The mixed EWMA-CUSUM control chart is discussed based on the covariate method, multiple measurements method, and linearly increasing variance method.

\subsection{Mixed EWMA-CUSUM control chart using covariates}

As discussed in Section $3, Y$ and $X$ are linearly correlated, i.e., $Y=A+B X+\varepsilon$, where $\varepsilon$ is random error and independent of $X, Y$ is normally distributed with mean $A+B \mu$ and variance $B^{2} \sigma^{2}+\sigma_{m}^{2}$, and it is assumed that $A, B, \sigma^{2}$ and $\sigma_{m}^{2}$ are known. Further, $Y$ is related to $X$ and $Y$ is distributed normally with mean $A+B \mu$ and its variance is $B^{2} \sigma^{2}+\sigma_{m}^{2}$. Then, we have:

$$
\begin{aligned}
& U_{i}=\lambda \bar{Y}_{i}+(1-\lambda) U_{i-1}, \\
& E\left(U_{i}\right)=A+B \mu, \\
& \sigma^{2}\left(U_{i}\right)=\left(\frac{\lambda}{2-\lambda}\right)\left(1-(1-\lambda)^{2 i}\right)\left(\frac{B^{2} \sigma^{2}+\sigma_{m}^{2}}{n}\right),
\end{aligned}
$$

where $\bar{Y}_{i}$ is the mean value at time $i=1,2,3, \ldots$ and $\lambda$ is a constant that lies between 0 and 1 . $A$ and $B$ are two known constants. The statistics for the mixed EWMACUSUM control chart using covariates are given by:

$$
R_{i}^{+}=\max \left[0,\left(U_{i}-\mu\right)-K_{c}^{\prime \prime}+R_{i-1}^{+}\right],
$$




$$
R_{i}^{-}=\max \left[0,-\left(U_{i}-\mu\right)-K_{c}^{\prime \prime}+R_{i-1}^{-}\right]
$$

where the control limit is denoted by $H_{c}^{\prime \prime}$. The process mean is said to be shifted above the target value if $R_{i}^{+}>H_{c}^{\prime \prime}$ or said to be shifted below the target value if $R_{i}^{-}>H_{c}^{\prime}$.

\subsection{Mixed EWMA-CUSUM chart by using multiple measurements}

For more precise results, Linna and Woodall [8] suggested multiple measurements rather than one measurement in each sample. They concluded that as the number of multiple measurements increases, the variance of error component in the average of multiple observations becomes smaller. In fact, if the multiple measurements approach infinity, then the variance will approach zero. In this paper, the effect of multiple measurements on the mixed EWMA-CUSUM chart has been studied. Here, it is assumed that for each sampling point, there are $p$ measurements for each value of $n$ observations.

Again, this study uses the statistic for multiple measurements with a mean of $A+B \mu$.

$$
U_{i}=\lambda \bar{Y}_{i}+(1-\lambda) U_{i-1}
$$

and variance is given as:

$$
\operatorname{var}\left(U_{i}\right)=\left(\frac{\lambda}{2-\lambda}\right)\left(1-(1-\lambda)^{2 i}\right)\left(\frac{B^{2} \sigma^{2}}{n}+\frac{\sigma_{t}^{2}}{n p}\right) .
$$

The statistics for mixed EWMA-CUSUM chart are given by:

$$
\begin{aligned}
& R_{i p}^{+}=\max \left[0,-\left(U_{i}-\mu\right)-K_{c}^{\prime \prime \prime}+R_{i p-1}^{-}\right], \\
& R_{i p}^{-}=\max \left[0,-\left(U_{i}-\mu\right)-K_{c}^{\prime \prime \prime}+R_{i p-1}^{-}\right],
\end{aligned}
$$

where $K$ is the reference value and the control limit for the given statistics is denoted by $H_{c}^{\prime \prime}$. The process mean is said to be shifted above the target value if $R_{i p}^{+}>H_{c}^{\prime \prime \prime}$ and said to be shifted below the target value if $R_{i p}^{-}>H_{c}^{\prime \prime \prime}$.

\subsection{Mixed EWMA-CUSUM with linearly increasing variance}

This section studies the effect of linearly increasing variance on the mixed EWMA-CUSUM chart. It is assumed that the variance linearly changes with $X$ and the statistic used for linearly increasing variance with mean $A+B \mu$ is defined as follows:

$$
U_{i}=\lambda \bar{Y}_{i}+(1-\lambda) U_{i-1},
$$

and the variance is given by:

$$
\begin{aligned}
\operatorname{var}\left(U_{i}\right) & =\left(\frac{\lambda}{2-\lambda}\right) \\
& \left(1-(1-\lambda)^{2 i}\right)\left(\frac{B^{2} \sigma^{2}+C+D \mu}{n}\right) .
\end{aligned}
$$

After using the EWMA statistic, the upper-sided and lower-sided statistics are given as follows:

$$
\begin{aligned}
& R_{i q}^{+}=\max \left[0,-\left(U_{i}-\mu\right)-K_{c}^{\prime}+R_{i q-1}^{-}\right], \\
& R_{i q}^{-}=\max \left[0,-\left(U_{i}-\mu\right)-K_{c}^{\prime}+R_{i q-1}^{-}\right] .
\end{aligned}
$$

The given statistics are plotted against the control limit, denoted by $H_{c}^{\prime}$. If the statistic $R_{i q}^{-}$is plotted above $H_{c}^{\prime}$, the process mean is said to be shifted below the target value; and if $R_{i q}^{+}$is plotted above $H_{c}^{\prime}$, then the process mean is shifted above the target value.

\section{Effect of measurement error}

The CUSUM and EWMA control charts are based on past information along with current information being the reason why they are known as memory-type control charts. Due to this feature, these charts are more efficient to detect small and moderate shifts (cf., Abbas [19]). In this paper, the EWMA statistic is used to find the CUSUM statistic called mixed EWMA-CUSUM control chart. To observe the effect of measurement error on this chart, we have fixed the in-control Average Run Length (ARL) at 500 where $\lambda=0.1$. The ARLs and Standard Deviation of Run Length (SDRLs) for the mixed EWMA-CUSUM chart with the covariates model are presented in Table 1 for $n=1,3,5$ using different values of error ratio $\left(\frac{\sigma_{m}^{2}}{\sigma^{2}}\right)$ taken as $0.1,0.2$, $0.3,0.5$, and 1. According to Table 1, the ARLs are minimum when there is no measurement error. In case a measurement error is added, the ARL increases as the error ratio increases. For example, at shift 0.25, the ARL is 79.846 in case of no measurement error. Given the addition of the measurement error in the form of error ratio from 0.1 to 1 , the ARLs are 85.083, 90.093, 94.836, 105.073, and 126.292. Further, we observed the effect of sample size. As in Table 1, the ARL is 85.083 with an error ratio of 0.1 and $n=1$ at a shift of 0.25 . Moreover, at the same shift with sample size $n=3$, the value of ARL is reduced to 20.362. When $n=5$, the ARL is 10.987 which indicates that as the sample size increases, the negative effect of measurement error is reduced. Table 2 chooses different values of $B=1,2,3,5$ where $A=0$ and the ratio $\frac{\sigma_{m}^{2}}{\sigma^{2}}=1$. According to the results in Table 2, the ARLs are decreasing following an increase in the value of $B$. The result is similar to that obtained by Maravelakis et al. [9]. For example, at shift 0.5, the ARLs are $52.119,36.394,35.768$, and 35.608 when $B=1,2,3$, and 5 , respectively. The value of $B$ contributes to the reduction of the adverse effect of measurement error. Table 3 provides the ARLs with multiple measurements taking $p=5$ and $B=1$ with different error ratio values. From Table 3, it is concluded that the effect 
Table 1. The ARL (SDRL) of the proposed chart with covariates using $k=0.5, \lambda=0.1$ and different values of $\frac{\sigma_{m}^{2}}{\sigma^{2}}$.

\begin{tabular}{|c|c|c|c|c|c|c|}
\hline $\begin{array}{c}\text { Shift } \\
(n=1)\end{array}$ & WOE* & 0.1 & 0.2 & 0.3 & 0.5 & 1 \\
\hline 0 & $501.039(463.201)$ & $500.023(462.465)$ & $500.071(461.297)$ & $497.850(456.189)$ & $500.271(462.155)$ & $498.537(458.307)$ \\
\hline 0.25 & $79.846(48.771)$ & $85.083(53.254)$ & $90.093(57.706)$ & $94.836(62.750)$ & $105.073(71.155)$ & $126.292(91.574)$ \\
\hline 0.5 & $35.518(13.021)$ & $37.341(14.172)$ & $38.951(15.234)$ & $40.788(16.687)$ & $43.827(18.712)$ & $52.119(25.226)$ \\
\hline 1 & $18.867(3.806)$ & $19.614(4.110)$ & $20.274(4.385)$ & $21.032(4.702)$ & $22.311(5.332)$ & $25.340(6.839)$ \\
\hline 1.5 & $13.791(2.038)$ & $14.292(2.181)$ & $14.758(2.328)$ & $15.206(2.479)$ & $16.066(2.742)$ & $17.957(3.449)$ \\
\hline 2 & $11.194(1.352)$ & $11.588(1.450)$ & $11.938(1.533)$ & $12.292(1.621)$ & $12.929(1.792)$ & $14.404(2.216)$ \\
\hline 2.5 & $9.556(1.009)$ & $9.880(1.072)$ & 10.190(1.139) & $10.469(1.187)$ & $11.026(1.317)$ & $12.234(1.604)$ \\
\hline 3 & $8.412(0.806)$ & $8.698(0.852)$ & $8.958(0.898)$ & $9.219(0.946)$ & $9.689(1.037)$ & $10.727(1.254)$ \\
\hline \multicolumn{7}{|c|}{ Sample size $=\mathbf{3}$} \\
\hline Shift & WOE & 0.1 & 0.2 & 0.3 & 0.5 & 1 \\
\hline 0 & $503.038(481.905)$ & $502.632(481.130)$ & $500.773(478.594)$ & $506.145(484.818)$ & $503.522(481.129)$ & $506.453(484.849)$ \\
\hline 0.25 & $19.366(6.408)$ & $20.362(7.033)$ & $21.271(7.649)$ & $22.227(8.229)$ & $24.061(9.537)$ & $28.365(12.799)$ \\
\hline 0.5 & $10.369(1.958)$ & $10.789(2.116)$ & $11.174(2.238)$ & $11.564(2.405)$ & $12.279(2.688)$ & 13.934(3.3887) \\
\hline 1 & $6.062(0.746)$ & $6.284(0.789)$ & $6.492(0.842)$ & $6.694(0.884)$ & $7.061(0.973)$ & $7.885(1.176)$ \\
\hline 1.5 & $4.491(0.523)$ & $4.661(0.535)$ & $4.819(0.534)$ & $4.965(0.543)$ & $5.220(0.585)$ & $5.803(0.700)$ \\
\hline 2 & $3.718(0.455)$ & $3.858(0.376)$ & $3.954(0.323)$ & $4.034(0.332)$ & $4.206(0.428)$ & $4.698(0.537)$ \\
\hline 2.5 & $3.031(0.175)$ & $3.098(0.297)$ & $3.211(0.408)$ & $3.360(0.480)$ & $3.644(0.481)$ & $4.013(0.323)$ \\
\hline 3 & $2.950(0.217)$ & $2.986(0.115)$ & $2.998(0.066)$ & $3.005(0.083)$ & $3.052(0.222)$ & $3.497(0.500)$ \\
\hline \multicolumn{7}{|c|}{ Sample size $=5$} \\
\hline Shift & WOE & 0.1 & 0.2 & 0.3 & 0.5 & 1 \\
\hline 0 & $503.206(487.171)$ & $499.360(482.750)$ & $502.553(486.428)$ & $504.589(491.073)$ & $502.721(486.614)$ & $507.123(490.720)$ \\
\hline 0.25 & $10.333(2.620)$ & $10.798(2.881)$ & $11.26(3.063)$ & $11.702(3.299)$ & $12.585(3.748)$ & $14.597(4.899)$ \\
\hline 0.5 & $5.688(0.923)$ & $5.900(0.982)$ & $6.120(1.047)$ & $6.327(1.102)$ & $6.713(1.225)$ & $7.587(1.518)$ \\
\hline 1 & $3.256(0.441)$ & $3.392(0.495)$ & $3.529(0.519)$ & $3.652(0.521)$ & $3.869(0.514)$ & $4.310(0.585)$ \\
\hline 1.5 & $2.354(0.478)$ & $2.537(0.498)$ & $2.698(0.458)$ & $2.806(0.396)$ & $2.938(0.263)$ & $3.120(0.340)$ \\
\hline 2 & $2.000(0.010)$ & $2.000(0.030)$ & $2.004(0.069)$ & $2.018(0.134)$ & $2.103(0.304)$ & $2.582(0.493)$ \\
\hline 2.5 & $1.987(0.110)$ & $1.997(0.052)$ & $1.999(0.021)$ & $1.999(0.013)$ & $2.000(0.004)$ & $2.014(0.120)$ \\
\hline 3 & $1.400(0.490)$ & $1.667(0.471)$ & $1.850(0.356)$ & $1.943(0.230)$ & $1.993(0.081)$ & $2(0)$ \\
\hline
\end{tabular}

${ }^{*} \mathrm{WOE}=$ Without Error

of measurement error on the mixed EWMA-CUSUM control chart increases with increase in error ratio. For example, in Table 3, at shift 0.25 , the ARL increases from 79.846 to 90.052 as the error ratio increases from 0.1 to 1 . In Table 4 , different values of $B$ are considered and the results are compared. ARL is minimum at large values of $B$, meaning that the effect of measurement error is reduced with an increase in $B$. For example, in Table 4, the ARLs decrease from 90.052 to 80.784 with an increase in the value of $B$ from 1 to 5. The ARL (SDRL) for the mixed EWMA-CUSUM chart with multiple measurements is presented in Table 5 with different values of $p=5,10,20,50$. According to Table 5 , the adverse effect of measurement error is 
Table 2. The ARL (SDRL) of the proposed chart with covariates for different values of $B$.

\begin{tabular}{|c|c|c|c|c|c|}
\hline $\begin{array}{c}\text { Shift } \\
(n=1)\end{array}$ & WOE & 1 & 2 & 3 & 5 \\
\hline 0 & $501.039(463.201)$ & $498.537(458.307)$ & $498.050(461.601)$ & $500.468(461.514)$ & $501.115(461.529)$ \\
\hline 0.25 & $79.846(48.771)$ & $126.292(91.574)$ & $82.699(51.544)$ & $80.914(49.645)$ & $80.141(49.038)$ \\
\hline 0.5 & $35.518(13.021)$ & $52.119(25.226)$ & $36.394(13.659)$ & $35.7628(13.097)$ & $35.608(13.093)$ \\
\hline 1 & $18.867(3.806)$ & $25.340(6.839)$ & $19.243(3.961)$ & $19.015(3.851)$ & $18.913(3.826)$ \\
\hline 1.5 & $13.791(2.038)$ & $17.957(3.449)$ & $14.046(2.111)$ & $13.919(2.085)$ & $13.827(2.044)$ \\
\hline 2 & $11.194(1.352)$ & $14.40(2.216)$ & $11.378(1.402)$ & $11.281(1.373)$ & $11.229(1.363)$ \\
\hline 2.5 & $9.556(1.009)$ & $12.234(1.604)$ & $9.711(1.039)$ & $9.624(1.017)$ & $9.577(1.022)$ \\
\hline 3 & $8.412(0.806)$ & $10.727(1.254)$ & $8.557(0.826)$ & $8.475(0.812)$ & $8.438(0.803)$ \\
\hline \multicolumn{6}{|c|}{ Sample size $=3$} \\
\hline Shift & WOE & 1 & 2 & 3 & 5 \\
\hline 0 & $503.038(481.905)$ & $506.453(484.849)$ & $501.372(484.528)$ & $505.958(483.229)$ & $502.081(480.795)$ \\
\hline 0.25 & $19.366(6.408)$ & $28.365(12.799)$ & $21.712(7.879)$ & $20.460(7.059)$ & $19.715(6.614)$ \\
\hline 0.5 & $10.369(1.958)$ & $13.934(3.3887)$ & $11.391(2.330)$ & $10.838(2.113)$ & $10.549(2.015)$ \\
\hline 1 & $6.062(0.746)$ & $7.885(1.176)$ & $6.588(0.864)$ & $6.309(0.798)$ & $6.151(0.766)$ \\
\hline 1.5 & $4.491(0.523)$ & $5.803(0.700)$ & $4.892(0.535)$ & $4.681(0.534)$ & $4.562(0.533)$ \\
\hline 2 & $3.718(0.455)$ & $4.698(0.537)$ & $3.991(0.323)$ & $3.869(0.369)$ & $3.783(0.421)$ \\
\hline 2.5 & $3.031(0.175)$ & $4.013(0.323)$ & $3.283(0.450)$ & $3.107(0.309)$ & $3.053(0.226)$ \\
\hline 3 & $2.950(0.217)$ & $3.497(0.500)$ & $3.001(0.068)$ & $2.989(0.105)$ & $2.970(0.169)$ \\
\hline \multicolumn{6}{|c|}{ Sample size $=\mathbf{5}$} \\
\hline Shift & WOE & 1 & 2 & 3 & 5 \\
\hline 0 & $503.206(487.171)$ & $507.123(490.720)$ & $500.079(483.880)$ & $506.501(489.679)$ & $502.491(491.965)$ \\
\hline 0.25 & $10.333(2.620)$ & $14.597(4.899)$ & $11.471(3.196)$ & $10.848(2.876)$ & $10.526(2.718)$ \\
\hline 0.5 & $5.688(0.923)$ & $7.587(1.518)$ & $6.234(1.076)$ & $5.930(0.989)$ & $5.767(0.942)$ \\
\hline 1 & $3.256(0.441)$ & $4.310(0.585)$ & $3.593(0.520)$ & $3.408(0.499)$ & $3.308(0.466)$ \\
\hline 1.5 & $2.3542(0.478)$ & $3.120(0.340)$ & $2.755(0.430)$ & $2.554(0.497)$ & $2.431(0.495)$ \\
\hline 2 & $2.000(0.010)$ & $2.582(0.493)$ & $2.010(0.101)$ & $2.001(0.033)$ & $2.000(0.014)$ \\
\hline 2.5 & $1.987(0.110)$ & $2.014(0.120)$ & $1.999(0.015)$ & $1.997(0.045)$ & $1.993(0.083)$ \\
\hline 3 & $1.400(0.490)$ & $2(0)$ & $1.902(0.296)$ & $1.694(0.460)$ & $1.512(0.499)$ \\
\hline
\end{tabular}

reduced as $p$ increases. For example, the ARLs decrease from 90.052 to 81.501 when $p$ increases from 5 to 50 .

Table 6 presents the effect of linearly increasing variance for different values of $D=1,2,3,5$ with $C=0$. In linearly increasing variance, the effect of error variance is changing frequently due to the factor
$D_{\mu}$. The ARLs are increasing with an increase in $D$. For example, at shift 0.25 , the ARLs are 310.69, $378.116,409.311$, and 439.118 when $D=1,2,3$, and 5 , respectively. In Table 7 , the effect of linearly increasing variance is evaluated at different values of $C$, i.e., $C=0,1,2,3$. According to Table 7 , ARL values are 
Table 3. The ARL (SDRL) of the proposed chart with multiple measurements for different values of $\frac{\sigma_{m}^{2}}{\sigma^{2}}$ when $p=5$, $B=1$

\begin{tabular}{|c|c|c|c|c|c|c|}
\hline $\begin{array}{c}\text { Shift } \\
(n=1)\end{array}$ & WOE & 0.1 & 0.2 & 0.3 & 0.5 & 1 \\
\hline 0 & $501.039(463.20)$ & $501.626(462.45)$ & $499.630(459.76)$ & $504.583(464.20)$ & $498.985(462.61)$ & $498.252(457.827)$ \\
\hline 0.25 & $79.846(48.771)$ & $80.947(49.659)$ & $82.091(50.988)$ & $82.599(51.595)$ & $85.962(53.831)$ & $90.052(57.715)$ \\
\hline 0.5 & $35.518(13.021)$ & $35.748(13.167)$ & $36.313(13.415)$ & $36.552(13.750)$ & $37.239(14.149)$ & $38.991(15.305)$ \\
\hline 1 & $18.867(3.806)$ & $19.018(3.849)$ & $19.129(3.906)$ & $19.322(4.002)$ & $19.630(4.125)$ & $20.339(4.420)$ \\
\hline 1.5 & $13.791(2.038)$ & $13.883(2.047)$ & $13.988(2.102)$ & $14.098(2.130)$ & $14.303(2.188)$ & $14.779(2.347)$ \\
\hline 2 & $11.194(1.352)$ & $11.263(1.373)$ & $11.331(1.389)$ & $11.424(1.410)$ & $11.578(1.447)$ & $11.937(1.535)$ \\
\hline 2.5 & $9.556(1.009)$ & $9.616(1.018)$ & $9.688(1.036)$ & $9.755(1.048)$ & $9.879(1.067)$ & $10.181(1.146)$ \\
\hline 3 & $8.412(0.806)$ & $8.476(0.815)$ & $8.524(0.821)$ & $8.585(0.831)$ & $8.691(0.847)$ & $8.966(0.901)$ \\
\hline \multicolumn{7}{|c|}{ Sample size $=3$} \\
\hline Shift & WOE & 0.1 & 0.2 & 0.3 & 0.5 & 1 \\
\hline 0 & $503.038(481.905)$ & $504.491(487.2)$ & $505.190(483.97)$ & $501.593(478.42)$ & $504.620(479.09)$ & $504.076(484.483)$ \\
\hline 0.25 & $19.366(6.408)$ & $19.549(6.503)$ & $19.673(6.611)$ & $19.925(6.730)$ & $20.344(7.005)$ & $21.275(7.573)$ \\
\hline 0.5 & $10.369(1.958)$ & $10.465(1.996)$ & $10.544(2.015)$ & $10.618(2.039)$ & $10.801(2.105)$ & $11.175(2.250)$ \\
\hline 1 & $6.062(0.746)$ & $6.113(0.758)$ & $6.145(0.766)$ & $6.198(0.776)$ & $6.278(0.793)$ & $6.494(0.836)$ \\
\hline 1.5 & $4.491(0.523)$ & $4.524(0.528)$ & $4.561(0.532)$ & $4.595(0.532)$ & $4.662(0.535)$ & $4.816(0.536)$ \\
\hline 2 & $3.718(0.455)$ & $3.751(0.439)$ & $3.783(0.421)$ & $3.811(0.404)$ & $3.854(0.379)$ & $3.953(0.323)$ \\
\hline 2.5 & $3.031(0.175)$ & $3.040(0.197)$ & $3.052(0.222)$ & $3.066(0.249)$ & $3.100(0.300)$ & $3.213(0.409)$ \\
\hline 3 & $2.950(0.217)$ & $2.963(0.188)$ & $2.970(0.1682)$ & $2.977(0.147)$ & $2.987(0.112)$ & $2.997(0.067)$ \\
\hline \multicolumn{7}{|c|}{ Sample size $=5$} \\
\hline Shift & WOE & 0.1 & 0.2 & 0.3 & 0.5 & 1 \\
\hline 0 & $503.206(487.17)$ & $503.797(493.1)$ & $501.614(485.77)$ & $500.566(484.07)$ & $498.949(483.71)$ & $503.575(488.481)$ \\
\hline 0.25 & $10.333(2.620)$ & $10.436(2.697)$ & $10.525(2.725)$ & $10.619(2.788)$ & $10.813(2.874)$ & $11.264(3.076)$ \\
\hline 0.5 & $5.688(0.923)$ & $5.726(0.934)$ & $5.773(0.945)$ & $5.822(0.957)$ & $5.898(0.977)$ & $6.122(1.049)$ \\
\hline 1 & $3.256(0.441)$ & $3.279(0.454)$ & $3.307(0.467)$ & $3.333(0.477)$ & $3.388(0.494)$ & $3.532(0.518)$ \\
\hline 1.5 & $2.3542(0.47827)$ & $2.393(0.488)$ & $2.433(0.495)$ & $2.467(0.498)$ & $2.538(0.498)$ & $2.695(0.460)$ \\
\hline 2 & $2.00012(0.0109)$ & $2.000(0.014)$ & $2.000(0.016)$ & $2.000(0.017)$ & $2.000(0.029)$ & $2.004(0.070)$ \\
\hline 2.5 & $1.987(0.110)$ & $1.991(0.094)$ & $1.993(0.079)$ & $1.994(0.072)$ & $1.997(0.051)$ & $1.999(0.018)$ \\
\hline 3 & $1.400(0.490)$ & $1.456(0.498)$ & $1.510(0.499)$ & $1.566(0.495)$ & $1.666(0.471)$ & $1.852(0.354)$ \\
\hline
\end{tabular}

maximum at a large value of $C$. For example, at shift 0.25, the ARLs are $310.69319 .63,328.115$, and 338.269 when $C=0,1,2$, and 3 , respectively. The ARLs in Tables 1 and 3 are the same if $p=1$.

\section{Comparative results}

This study considered an existing control chart named mixed EWMA-CUSUM (cf., Abbas et al. [3]) under the situation of measurement error. Tables $1-7$ provide the effect of measurement error on the existing chart. According to ARLs, the mixed EWMA-CUSUM control chart is adversely affected by measurement error. Maravelakis [11] concluded that the CUSUM chart was the best for a small shift and the EWMA was the best for a large shift in the presence of measurement 
Table 4. The ARL (SDRL) of the proposed chart for multiple measurements with $p=5, \frac{\sigma_{m}^{2}}{\sigma^{2}}=1$, and different values of B.

\begin{tabular}{|c|c|c|c|c|c|}
\hline $\begin{array}{c}\text { Shift } \\
(n=1)\end{array}$ & WOE & 1 & 2 & 3 & 5 \\
\hline 0 & $501.039(463.201)$ & $498.252(457.827)$ & $499.306(461.703)$ & $498.805(458.129)$ & $503.309(460.941)$ \\
\hline 0.25 & $79.846(48.771)$ & $90.052(57.715)$ & $82.848(51.437)$ & $81.276(49.734)$ & $80.748(49.306)$ \\
\hline 0.5 & $35.518(13.021)$ & $38.991(15.305)$ & $36.385(13.642)$ & $35.802(13.152)$ & $35.590(12.934)$ \\
\hline 1 & $18.867(3.806)$ & $20.339(4.420)$ & $19.210(3.935)$ & $19.002(3.861)$ & $18.911(3.811)$ \\
\hline 1.5 & $13.791(2.038)$ & $14.779(2.347)$ & $14.062(2.116)$ & $13.890(2.057)$ & $13.844(2.040)$ \\
\hline 2 & $11.194(1.352)$ & $11.937(1.535)$ & $11.381(1.394)$ & $11.280(1.368)$ & $11.224(1.355)$ \\
\hline 2.5 & $9.556(1.009)$ & $10.181(1.146)$ & $9.716(1.039)$ & $9.626(1.023)$ & $9.576(1.013)$ \\
\hline 3 & $8.412(0.806)$ & $8.966(0.901)$ & $8.55352(0.822)$ & $8.476(0.816)$ & $8.440(0.808)$ \\
\hline
\end{tabular}

Sample size $=3$

\begin{tabular}{cccccc}
\hline Shift & WOE & $\mathbf{1}$ & $\mathbf{2}$ & $\mathbf{3}$ & $\mathbf{5}$ \\
\hline 0 & $503.038(481.905)$ & $504.076(484.483)$ & $502.644(477.281)$ & $506.997(481.392)$ & $503.610(482.150)$ \\
0.25 & $19.366(6.408)$ & $21.275(7.573)$ & $19.818(6.662)$ & $19.575(6.501)$ & $19.469(6.438)$ \\
0.5 & $10.369(1.958)$ & $11.175(2.250)$ & $10.583(2.018)$ & $10.467(1.983)$ & $10.392(1.963)$ \\
1 & $6.062(0.746)$ & $6.494(0.836)$ & $6.167(0.774)$ & $6.112(0.761)$ & $6.081(0.753)$ \\
1.5 & $4.491(0.523)$ & $4.816(0.536)$ & $4.575(0.534)$ & $4.530(0.530)$ & $4.49(0.525)$ \\
2 & $3.718(0.455)$ & $3.953(0.323)$ & $3.795(0.415)$ & $3.757(0.436)$ & $3.73(0.448)$ \\
2.5 & $3.031(0.175)$ & $3.213(0.409)$ & $3.060(0.237)$ & $3.043(0.204)$ & $3.034(0.182)$ \\
3 & $2.950(0.217)$ & $2.997(0.067)$ & $2.975(0.155)$ & $2.964(0.185)$ & $2.957(0.202)$ \\
\hline
\end{tabular}

Sample size $=\mathbf{5}$

\begin{tabular}{cccccc}
\hline Shift & WOE & $\mathbf{1}$ & $\mathbf{2}$ & $\mathbf{3}$ & $\mathbf{5}$ \\
\hline 0 & $503.206(487.171)$ & $503.575(488.481)$ & $502.797(489.745)$ & $501.401(486.2455)$ & $502.999(485.234)$ \\
0.25 & $10.333(2.620)$ & $11.264(3.076)$ & $10.5648(2.738)$ & $10.440(2.668)$ & $10.378(2.653)$ \\
0.5 & $5.688(0.923)$ & $6.122(1.049)$ & $5.795(0.950)$ & $5.734(0.937)$ & $5.705(0.928)$ \\
1 & $3.256(0.441)$ & $3.532(0.518)$ & $3.320(0.471)$ & $3.284(0.455)$ & $3.266(0.446)$ \\
1.5 & $2.354(0.478)$ & $2.695(0.460)$ & $2.445(0.497)$ & $2.398(0.489)$ & $2.373(0.483)$ \\
2 & $2.000(0.010)$ & $2.004(0.070)$ & $2.000(0.020)$ & $2.000(0.016)$ & $2.000(0.010)$ \\
3 & $1.987(0.110)$ & $1.999(0.018)$ & $1.994(0.073)$ & $1.991(0.089)$ & $1.989(0.101)$ \\
\hline
\end{tabular}

error. Here, a reasonable question is which one of the three (EWMA, CUSUM, and mixed EWMA-CUSUM) control charts has the best performance in terms of ARLs with measurement error. In Tables 8 and 9 , there are three different control charts with out-of-control cases. Given that we cannot compare them directly, we follow the procedure described by Maravelakis [11] for comparisons and find the percentage increase for each specific shift in the no-error case. For example, in Table 8, the ARLs for the CUSUM control chart at 
Table 5. The ARL (SDRL) of the proposed chart with multiple measurements for different values of $p$ where $B=1$.

\begin{tabular}{cccccc}
\hline $\begin{array}{c}\text { Shift } \\
(\boldsymbol{n}=\mathbf{1})\end{array}$ & WOE & $\mathbf{5}$ & $\mathbf{1 0}$ & $\mathbf{2 0}$ & $\mathbf{5 0}$ \\
\hline 0 & $501.039(463.201)$ & $498.252(457.827)$ & $498.611(459.308)$ & $500.840(461.226)$ & $499.021(459.55)$ \\
0.25 & $79.846(48.771)$ & $90.052(57.715)$ & $85.060(53.639)$ & $82.781(51.313)$ & $81.501(50.288)$ \\
0.5 & $35.518(13.021)$ & $38.991(15.305)$ & $37.259(14.146)$ & $36.301(13.472)$ & $35.639(13.048)$ \\
1 & $18.867(3.806)$ & $20.339(4.420)$ & $19.604(4.104)$ & $19.228(3.942)$ & $18.991(3.848)$ \\
1.5 & $13.791(2.038)$ & $14.779(2.347)$ & $14.282(2.184)$ & $14.047(2.110)$ & $13.882(2.054)$ \\
2 & $11.194(1.352)$ & $11.937(1.535)$ & $11.579(1.442)$ & $11.378(1.410)$ & $11.269(1.370)$ \\
2.5 & $9.556(1.009)$ & $10.181(1.146)$ & $9.875(1.072)$ & $9.7135(1.040)$ & $9.623(1.024)$ \\
3 & $8.412(0.806)$ & $8.966(0.901)$ & $8.697(0.853)$ & $8.556(0.826)$ & $8.473(0.813)$ \\
\hline
\end{tabular}

\begin{tabular}{|c|c|c|c|c|c|}
\hline \multicolumn{6}{|c|}{ Sample size $=3$} \\
\hline Shift & WOE & 5 & 10 & 20 & 50 \\
\hline 0 & $503.038(481.905)$ & $504.076(484.483)$ & $504.055(484.728)$ & $502.064(480.527)$ & $501.393(480.777)$ \\
\hline 0.25 & $19.366(6.408)$ & $21.275(7.5733)$ & $20.311(6.985)$ & $19.8775(6.694)$ & $19.549(6.517)$ \\
\hline 0.5 & $10.369(1.9586)$ & $11.175(2.250)$ & $10.785(2.092)$ & $10.598(2.040)$ & $10.466(1.990)$ \\
\hline 1 & $6.062(0.746)$ & $6.494(0.836)$ & $6.285(0.794)$ & $6.173(0.770)$ & $6.109(0.758)$ \\
\hline 1.5 & $4.491(0.523)$ & $4.816(0.536)$ & $4.662(0.537)$ & $4.575(0.532)$ & $4.523(0.527)$ \\
\hline 2 & $3.718(0.455)$ & $3.953(0.323)$ & $3.859(0.376)$ & $3.793(0.416)$ & $3.750(0.438)$ \\
\hline 2.5 & $3.031(0.175)$ & $3.213(0.409)$ & $3.099(0.298)$ & $3.059(0.236)$ & $3.041(0.199)$ \\
\hline 3 & $2.950(0.217)$ & $2.997(0.067)$ & $2.988(0.106)$ & $2.975(0.156)$ & $2.961(0.191)$ \\
\hline \multicolumn{6}{|c|}{ Sample size $=5$} \\
\hline Shift & WOE & 5 & 10 & 20 & 50 \\
\hline 0 & $503.206(487.171)$ & $503.575(488.481)$ & $500.362(487.338)$ & $502.593(486.900)$ & $498.798(481.032)$ \\
\hline 0.25 & $10.333(2.620)$ & $11.264(3.076)$ & $10.821(2.858)$ & $10.586(2.750)$ & $10.412(2.684)$ \\
\hline 0.5 & $5.688(0.923)$ & $6.122(1.049)$ & $5.902(0.983)$ & $5.794(0.955)$ & $5.726(0.933)$ \\
\hline 1 & $3.256(0.441)$ & $3.532(0.518)$ & $3.394(0.496)$ & $3.324(0.473)$ & $3.280(0.453)$ \\
\hline 1.5 & $2.354(0.478)$ & $2.695(0.460)$ & $2.536(0.498)$ & $2.444(0.496)$ & $2.390(0.487)$ \\
\hline 2 & $2.000(0.010)$ & $2.004(0.070)$ & $2.00088(0.029)$ & $2.000(0.019)$ & $2.000(0.009)$ \\
\hline 2.5 & $1.987(0.110)$ & $1.999(0.018)$ & $1.997(0.053)$ & $1.994(0.074)$ & $1.990(0.098)$ \\
\hline 3 & $1.400(0.490)$ & $1.852(0.354)$ & $1.667(0.471)$ & $1.536(0.498)$ & $1.456(0.498)$ \\
\hline
\end{tabular}

shift 0.25 are 150.36 and 144.89 for $\frac{\sigma_{m}^{2}}{\sigma^{2}}=0.1$, and the percentage increase in this case is:

$$
(150.36-144.89) \times 100 / 144.89=3.77 .
$$

At the same shift-and-error ratio, the corresponding percentage increase for EWMA is (116.57-106.51) $\times 100 / 10.52=9.44$, and similarly, it is (91.32-84.11) $\times 100 / 84.11=8.57$ for the mixed case. For the comparison, we have considered the differences between (mixed-CUSUM) and (mixed-EWMA) in Tables 10 and 11. According to Table 8, based on ARLs, it is revealed that the mixed EWMA-CUSUM control chart performs better than EWMA and CUSUM control 
Table 6. The ARL (SDRL) of the proposed chart with linearly increasing variance for different values of $D$ where $C=0$.

\begin{tabular}{|c|c|c|c|c|c|}
\hline $\begin{array}{c}\text { Shift } \\
(n=1)\end{array}$ & WOE & 1 & 2 & 3 & 5 \\
\hline 0 & $501.039(463.201)$ & $502.467(464.654)$ & 499.644(461.493) & $497.604(461.312)$ & $501.624(461.317)$ \\
\hline 0.25 & $79.846(48.771)$ & $310.69(274.398)$ & $378.116(337.478)$ & $409.311(372.418)$ & $439.118(401.713)$ \\
\hline 0.5 & $35.518(13.021)$ & $153.354(118.612)$ & $223.419(186.638)$ & $267.080(229.350)$ & $327.419(288.646)$ \\
\hline 1 & $18.867(3.806)$ & $63.063(34.011)$ & $95.113(61.717)$ & $122.629(88.396)$ & $168.112(132.242)$ \\
\hline 1.5 & $13.791(2.038)$ & $39.402(15.710)$ & $57.212(29.378)$ & $72.658(42.365)$ & $100.614(67.438)$ \\
\hline 2 & $11.194(1.352)$ & $29.460(9.1863)$ & $40.995(16.655)$ & $50.937(24.134)$ & $69.052(39.001)$ \\
\hline 2.5 & $9.556(1.009)$ & $23.931(6.142)$ & $32.376(10.917)$ & $39.679(15.847)$ & $52.704(25.876)$ \\
\hline 3 & $8.412(0.806)$ & $20.491(4.465)$ & $27.160(7.824)$ & $32.824(11.176)$ & $42.776(18.065)$ \\
\hline \multicolumn{6}{|c|}{ Sample size $=3$} \\
\hline Shift & WOE & 1 & 2 & 3 & 5 \\
\hline 0 & $503.038(481.905)$ & $503.727(487.825)$ & $508.789(487.636)$ & $504.486(481.252)$ & $501.640(478.155)$ \\
\hline 0.25 & $19.366(6.408)$ & $95.548(75.230)$ & $152.004(129.822)$ & $195.357(173.915)$ & $255.204(236.056)$ \\
\hline 0.5 & $10.369(1.958)$ & $34.793(18.001)$ & $54.628(35.820)$ & $73.543(53.549)$ & $106.714(85.596)$ \\
\hline 1 & $6.062(0.746)$ & $16.145(4.564)$ & $22.349(8.387)$ & $27.878(12.357)$ & $38.175(20.766)$ \\
\hline 1.5 & $4.491(0.523)$ & $11.283(2.290)$ & $14.928(3.908)$ & $17.962(5.540)$ & $23.243(8.956)$ \\
\hline 2 & $3.718(0.455)$ & $8.943(1.485)$ & $11.616(2.425)$ & $13.701(3.304)$ & $17.280(5.148)$ \\
\hline 2.5 & $3.031(0.175)$ & $7.502(1.077)$ & $9.670(1.714)$ & $11.345(2.315)$ & $14.067(3.479)$ \\
\hline 3 & $2.950(0.217)$ & $6.539(0.850)$ & $8.374(1.3117)$ & $9.756(1.740)$ & $12.007(2.588)$ \\
\hline \multicolumn{6}{|c|}{ Sample size $=5$} \\
\hline Shift & WOE & 1 & 2 & 3 & 5 \\
\hline 0 & $503.206(487.171)$ & $505.395(492.710)$ & $502.648(487.820)$ & $507.272(490.163)$ & $502.803(488.14)$ \\
\hline 0.25 & $10.333(2.620)$ & $44.475(30.069)$ & $74.514(58.732)$ & $101.503(85.373)$ & $147.029(131.416)$ \\
\hline 0.5 & $5.688(0.923)$ & $17.335(6.657)$ & $25.887(13.252)$ & $34.164(20.702)$ & $50.411(35.610)$ \\
\hline 1 & $3.256(0.441)$ & $8.719(1.941)$ & $11.757(3.305)$ & $14.319(4.740)$ & $18.915(7.819)$ \\
\hline 1.5 & $2.3542(0.478)$ & $6.162(1.053)$ & $8.111(1.714)$ & $9.664(2.346)$ & $12.202(3.560)$ \\
\hline 2 & $2.000(0.010)$ & $4.886(0.724)$ & $6.352(1.114)$ & $7.475(1.475)$ & $9.318(2.187)$ \\
\hline 2.5 & $1.987(0.110)$ & $4.109(0.538)$ & $5.293(0.818)$ & $6.201(1.070)$ & $7.641(1.535)$ \\
\hline 3 & $1.400(0.490)$ & $3.556(0.521)$ & $4.576(0.653)$ & $5.348(0.835)$ & $6.565(1.180)$ \\
\hline
\end{tabular}

charts in the presence of measurement error. Table 9 provides the ARLs of CUSUM, EWMA, and mixed EWMA-CUSUM control charts with multiple measurements using different values of $D$. Based on the small ARL values, we concluded that the performance of the mixed EWMA-CUSUM control chart was better than those of CUSUM and EWMA in all cases. Table 10 shows the differences in measurement error using the covariate model. According to this table, the differences are negative, implying that the mixed EWMA-CUSUM control chart has the smallest ARL compared to the EWMA and CUSUM. The mixed EWMA-CUSUM control chart provides better results at all shifts than EWMA and CUSUM; however, for CUSUM, the result is less efficient only at 0.25 shift. Table 11 provides the impact of measurement 
Table 7. The ARL (SDRL) of the proposed chart with linearly increasing variance for different values of $C$ where $D=1$ and $B=1$.

\begin{tabular}{|c|c|c|c|c|c|}
\hline $\begin{array}{c}\text { Shift } \\
(n=1)\end{array}$ & WOE & $\mathbf{0}$ & 1 & 2 & 3 \\
\hline 0 & $501.0393(463.201)$ & $502.467(464.654)$ & $503.015(462.476)$ & $502.536(463.147)$ & $496.419(458.603)$ \\
\hline 0.25 & $79.846(48.771)$ & $310.69(274.398)$ & $319.633(282.271)$ & $328.115(290.535)$ & $338.369(299.811)$ \\
\hline 0.5 & $35.518(13.021)$ & $153.354(118.612)$ & $162.161(125.372)$ & $169.066(132.939)$ & $178.241(141.877)$ \\
\hline 1 & $18.867(3.806)$ & $63.063(34.011)$ & $66.717(37.306)$ & $69.886(39.850)$ & $73.694(43.139)$ \\
\hline 1.5 & $13.791(2.038)$ & $39.402(15.710)$ & $41.233(16.973)$ & $43.222(18.424)$ & $44.974(19.724)$ \\
\hline 2 & $11.194(1.352)$ & $29.460(9.186)$ & $30.665(9.934)$ & $31.880(10.631)$ & $33.025(11.319)$ \\
\hline 2.5 & $9.556(1.009)$ & $23.931(6.142)$ & $24.866(6.582)$ & $25.841(7.068)$ & $26.724(7.586)$ \\
\hline 3 & $8.412(0.806)$ & $20.491(4.465)$ & $21.302(4.838)$ & $21.967(5.156)$ & $22.670(5.517)$ \\
\hline \multicolumn{6}{|c|}{ Sample size $=3$} \\
\hline Shift & WOE & 0 & 1 & 2 & 3 \\
\hline 0 & $503.038(481.905)$ & $503.727(487.825)$ & $503.886(482.649)$ & $504.153(482.875)$ & $503.964(485.715)$ \\
\hline 0.25 & $19.366(6.408)$ & $95.548(75.230)$ & $101.803(81.525)$ & $108.271(87.418)$ & $114.018(92.708)$ \\
\hline 0.5 & $10.369(1.958)$ & $34.793(18.001)$ & $36.768(19.775)$ & $38.996(21.580)$ & $41.090(23.672)$ \\
\hline 1 & $6.062(0.746)$ & $16.145(4.564)$ & $16.788(4.819)$ & $17.424(5.246)$ & $18.115(5.632)$ \\
\hline 1.5 & $4.491(0.523)$ & $11.283(2.290)$ & $11.697(2.445)$ & $12.091(2.616)$ & $12.473(2.761)$ \\
\hline 2 & $3.718(0.455)$ & $8.943(1.485)$ & $9.240(1.574)$ & $9.539(1.665)$ & $9.828(1.771)$ \\
\hline 2.5 & $3.031(0.175)$ & $7.502(1.077)$ & $7.763(1.148)$ & $8.007(1.205)$ & $8.241(1.279)$ \\
\hline 3 & $2.950(0.217)$ & $6.539(0.850)$ & $6.758(0.900)$ & $6.957(0.941)$ & $7.161(0.988)$ \\
\hline \multicolumn{6}{|c|}{ Sample size $=5$} \\
\hline Shift & WOE & $\mathbf{0}$ & 1 & 2 & 3 \\
\hline 0 & $503.206(487.171)$ & $505.395(492.710)$ & $503.827(488.952)$ & $501.782(488.747)$ & $506.231(487.559)$ \\
\hline 0.25 & $10.333(2.620)$ & $44.475(30.069)$ & $47.939(33.117)$ & $50.680(35.943)$ & $54.242(39.024)$ \\
\hline 0.5 & $5.688(0.923)$ & $17.335(6.657)$ & $18.220(7.3189)$ & $19.141(8.006)$ & $19.953(8.549)$ \\
\hline 1 & $3.256(0.441)$ & $8.719(1.941)$ & $9.056(2.075)$ & $9.410(2.228)$ & $9.717(2.385)$ \\
\hline 1.5 & $2.354(0.478)$ & $6.162(1.053)$ & $6.392(1.123)$ & $6.613(1.183)$ & $6.811(1.261)$ \\
\hline 2 & $2.000(0.010)$ & $4.886(0.724)$ & $5.060(0.767)$ & $5.226(0.805)$ & $5.385(0.848)$ \\
\hline 2.5 & $1.987(0.110)$ & $4.109(0.538)$ & $4.243(0.568)$ & $4.375(0.607)$ & $4.503(0.637)$ \\
\hline 3 & $1.400(0.490)$ & $3.556(0.521)$ & $3.694(0.520)$ & $3.814(0.514)$ & $3.923(0.514)$ \\
\hline
\end{tabular}

Table 8. ARLs for CUSUM, EWMA, and mixed EWMA-CUSUM control charts with different ratios of $\frac{\sigma_{m}^{2}}{\sigma^{2}}$ using the covariates method.

\begin{tabular}{|c|c|c|c|c|c|c|c|c|c|c|c|c|}
\hline \multirow[b]{2}{*}{ Shift } & \multicolumn{3}{|c|}{ No error } & \multicolumn{3}{|c|}{0.1} & \multicolumn{3}{|c|}{0.2} & \multicolumn{3}{|c|}{1} \\
\hline & CU & EW & EWCU & CU & EW & EWCU & CU & EW & EWCU & CU & EW & EWCU \\
\hline 0 & 500 & 500 & 502 & 500 & 500 & 506 & 500 & 500 & 503 & 500 & 500 & 505 \\
\hline 0.25 & 144.89 & 106.51 & 84.11 & 150.36 & 116.57 & 91.32 & 157.30 & 124.79 & 96.46 & 189.78 & 180.9 & 142.04 \\
\hline 0.5 & 38.87 & 31.32 & 30.88 & 41.47 & 34.08 & 32.82 & 43.97 & 36.89 & 34.85 & 61.55 & 58.40 & 50.27 \\
\hline 1 & 10.52 & 10.33 & 13.88 & 11.35 & 11.06 & 14.56 & 12.17 & 11.78 & 15.23 & 18.25 & 17.41 & 2012 \\
\hline 1.5 & 5.82 & 6.09 & 9.60 & 6.28 & 6.45 & 9.99 & 6.74 & 6.81 & 10.39 & 10.16 & 9.52 & 13.15 \\
\hline 2 & 4.06 & 4.36 & 7.59 & 4.37 & 4.60 & 7.87 & 4.69 & 4.83 & 8.14 & 7.04 & 6.54 & 10.09 \\
\hline 2.5 & 3.15 & 3.44 & 6.39 & 3.39 & 3.62 & 6.63 & 3.63 & 3.78 & 6.85 & 5.41 & 5.01 & 8.37 \\
\hline 3 & 2.60 & 2.87 & 5.59 & 2.79 & 3.01 & 5.78 & 2.98 & 3.14 & 5.98 & 4.42 & 4.09 & 7.24 \\
\hline
\end{tabular}


Table 9. ARLs for CUSUM, EWMA, and proposed chart with different values of $D$ using multiple measurements.

\begin{tabular}{|c|c|c|c|c|c|c|c|c|c|c|c|c|}
\hline \multirow[t]{2}{*}{ Shift } & \multicolumn{3}{|c|}{ No error } & \multicolumn{3}{|c|}{5} & \multicolumn{3}{|c|}{10} & \multicolumn{3}{|c|}{50} \\
\hline & CU & EW & EWCU & CU & EW & EWCU & CU & EW & EWCU & $\mathrm{CU}$ & EW & EWCU \\
\hline 0 & 500 & 500 & 500 & 500 & 500 & 500 & 500 & 500 & 500 & 500 & 500 & 500 \\
\hline 0.25 & 144.63 & 106.07 & 79.84 & 155.38 & 122.30 & 90.05 & 150.73 & 115.05 & 85.06 & 146.80 & 106.6 & 81.50 \\
\hline 0.5 & 38.84 & 31.04 & 35.52 & 43.62 & 36.89 & 38.99 & 41.68 & 34.05 & 37.2 & 39.85 & 31.65 & 35.63 \\
\hline 1 & 10.50 & 10.38 & 18.86 & 12.15 & 11.78 & 20.33 & 11.34 & 10.99 & 19.60 & 10.69 & 10.53 & 18.99 \\
\hline 1.5 & 5.82 & 6.08 & 13.79 & 6.72 & 6.77 & 14.77 & 6.28 & 6.43 & 14.28 & 5.92 & 6.16 & 13.88 \\
\hline 2 & 4.06 & 4.38 & 11.19 & 4.68 & 4.83 & 11.93 & 4.38 & 4.59 & 11.57 & 4.11 & 4.40 & 11.26 \\
\hline 2.5 & 3.15 & 3.44 & 9.55 & 3.62 & 3.77 & 10.18 & 3.37 & 3.60 & 9.87 & 3.20 & 3.46 & 9.62 \\
\hline 3 & 2.60 & 2.86 & 8.41 & 2.98 & 3.14 & 8.9 & 2.79 & 3.06 & 8.62 & 2.64 & 2.89 & 8.47 \\
\hline
\end{tabular}

Table 10. Comparison of CUSUM, EWMA, and proposed chart with covariates.

\begin{tabular}{ccccccccc}
\hline \multirow{2}{*}{ Shift } & \multicolumn{3}{c}{ Mixed-CUSUM } & \multicolumn{4}{c}{ Mixed-EWMA } \\
\cline { 2 - 9 } & $\mathbf{0 . 1}$ & $\mathbf{0 . 2}$ & $\mathbf{0 . 3}$ & $\mathbf{1}$ & $\mathbf{0 . 1}$ & $\mathbf{0 . 2}$ & $\mathbf{0 . 3}$ & $\mathbf{1}$ \\
\hline 0.25 & 4.8 & 6.12 & 11.9 & 37.89 & -0.87 & -2.48 & -0.18 & -0.6 \\
0.5 & -0.4 & -0.27 & 0.19 & 4.45 & -2.53 & -4.77 & -7.1 & -23.67 \\
1 & -2.99 & -5.96 & -8.71 & -28.52 & -2.17 & -4.31 & -6.42 & -23.58 \\
1.5 & -3.84 & -7.57 & -11.51 & -37.6 & -1.85 & -3.59 & -5.49 & -19.35 \\
2 & -3.95 & -8.26 & -27.00 & -40.46 & -1.82 & -3.53 & -5.12 & -17.07 \\
2.5 & -3.87 & -8.04 & -26.66 & -40.76 & -1.48 & -2.69 & -4.34 & -14.65 \\
3 & -3.91 & -7.65 & -25.96 & -40.4 & -1.48 & -2.43 & -3.74 & -13.02 \\
\hline
\end{tabular}

Table 11. Comparison of CUSUM, EWMA, and proposed chart using multiple measurements.

\begin{tabular}{ccccccc}
\hline \multirow{2}{*}{ Shift } & \multicolumn{3}{c}{ Mixed-CUSUM } & \multicolumn{3}{c}{ Mixed-EWMA } \\
\cline { 2 - 7 } & $\mathbf{5}$ & $\mathbf{1 0}$ & $\mathbf{5 0}$ & $\mathbf{5}$ & $\mathbf{1 0}$ & $\mathbf{5 0}$ \\
\hline 0.25 & 5.36 & 2.31 & 0.58 & -2.51 & -92.44 & -1.57 \\
0.5 & -2.54 & -2.44 & -2.3 & -9.08 & -4.85 & -1.66 \\
1 & -7.92 & -4.08 & -1.12 & -3.99 & -1.95 & -0.76 \\
1.5 & -8.36 & -4.38 & -1.07 & 0.33 & -2.23 & -0.67 \\
2 & -8.66 & -4.49 & -0.61 & 1.78 & -2.13 & -0.53 \\
2.5 & -8.33 & -3.63 & -0.85 & 2.82 & -1.3 & 0.15 \\
3 & -8.09 & -3.98 & -0.82 & 3.39 & -3.67 & -0.33 \\
\hline
\end{tabular}

error using the multiple measurements on CUSUM, EWMA, and mixed EWMA-CUSUM control charts. The percentage increase for each shift as compared to no measurement error case is presented in Table 10. For example, in Table 10, the ARLs for the CUSUM control chart at shift 0.25 are 155.38 and 144.63 for $\frac{\sigma_{m}^{2}}{\sigma^{2}}=$ 0.1 with $p=5$, and the percentage increase in this case is computed as $((155.38-144.63) \times 100 / 144.63)=$ 7.43 for CUSUM. At the same shift and error ratio, the corresponding percentage increase for EWMA is computed as $((122.30-106.07) \times 100 / 106.07)=15.30$ and similarly, the percentage increase for the mixed is $((90.05-79.84) \times 100 / 79.84)=12.78$. In Table 11 , the difference between (mixed-CUSUM) and (mixedEWMA) is made clear. According to Table 11, the mixed EWMA-CUSUM chart is providing the best results for a small shift compared to EWMA and CUSUM charts. The results are improved at all shifts except 0.25 shift.

\section{Application}

In this section, an example is provided with a real dataset. Tran et al. [20] considered a production process of $500(\mathrm{~mL})$ milk bottles and the quality characteristic of interest is the volume of each bottle. The parameters estimated from Phase I are $\mu=500.023$ and $\sigma=0.9616$. The first 10 subgroups are considered as in-control and the remaining are out-of-control. The data is available in Table 12 . The covariate model is assumed concerning parameters $A=0, B=1$, and error ratio $\frac{\sigma_{m}}{\sigma}=0.28$. Figures $1-3$ display the monitoring results of three control charts for the sample size $n=5$. Figure 1 presents the EWMA control chart with measurement error with $\lambda=0.1$ and $\mathrm{L}=1.26$ for $A R L_{0}=500$. It can be observed that the EWMA control chart triggers the out-of-control signals from \#13 to \#20. In Figure 2, with the upper-sided CUSUM control chart with measurement error using $k^{+}=0.05$ and $h^{+}=1.45$, the out-of-control signals are triggered 
Table 12. Data set from Tran et al. [20].

\begin{tabular}{ccccccc}
\hline $\boldsymbol{i}$ & $\boldsymbol{X}_{\boldsymbol{i}, \boldsymbol{1}}$ & $\boldsymbol{X}_{\boldsymbol{i}, \boldsymbol{2}}$ & $\boldsymbol{X}_{\boldsymbol{i}, \boldsymbol{3}}$ & $\boldsymbol{X}_{\boldsymbol{i}, \boldsymbol{4}}$ & $\boldsymbol{X}_{\boldsymbol{i}, \boldsymbol{5}}$ & Mean \\
\hline 1 & 500.46 & 498.99 & 500.22 & 500.41 & 498.96 & 499.808 \\
2 & 500.06 & 500.02 & 499.31 & 501.07 & 499.57 & 500.042 \\
3 & 498.82 & 501.55 & 499.48 & 499.2 & 501.56 & 500.122 \\
4 & 502.64 & 502.86 & 500.06 & 499.08 & 500.72 & 501.072 \\
5 & 500.06 & 500.03 & 500.09 & 498.88 & 497.64 & 499.34 \\
6 & 500.5 & 499.54 & 499.02 & 498.09 & 499.87 & 499.32 \\
7 & 498.89 & 500.2 & 501.1 & 502.01 & 500.99 & 500.638 \\
8 & 500.37 & 499.28 & 500.15 & 500.87 & 500.88 & 500.31 \\
9 & 499.81 & 500.62 & 500.68 & 500.67 & 500 & 500.35 \\
10 & 499.79 & 499.87 & 500.98 & 499.12 & 500.79 & 500.11 \\
11 & 502.39 & 500.61 & 501.29 & 500.32 & 500.74 & 501.07 \\
12 & 500.35 & 500.57 & 501.8 & 502.03 & 499.56 & 500.86 \\
13 & 499.49 & 501.09 & 499.53 & 499.82 & 499.9 & 499.96 \\
14 & 500.96 & 500.87 & 502.71 & 500.7 & 499.71 & 500.96 \\
15 & 500.85 & 499.88 & 500.58 & 501.62 & 501.04 & 500.79 \\
16 & 500.48 & 502.82 & 501 & 501.78 & 501.73 & 501.56 \\
17 & 502.01 & 501.18 & 500.67 & 501.31 & 499.98 & 501.03 \\
18 & 501.36 & 501.11 & 500.27 & 501.12 & 501.98 & 501.168 \\
19 & 499.92 & 500.13 & 501.46 & 502.29 & 502.78 & 501.316 \\
20 & 501.19 & 500.3 & 499.03 & 500.17 & 502.19 & 500.576 \\
\hline & & & & & &
\end{tabular}

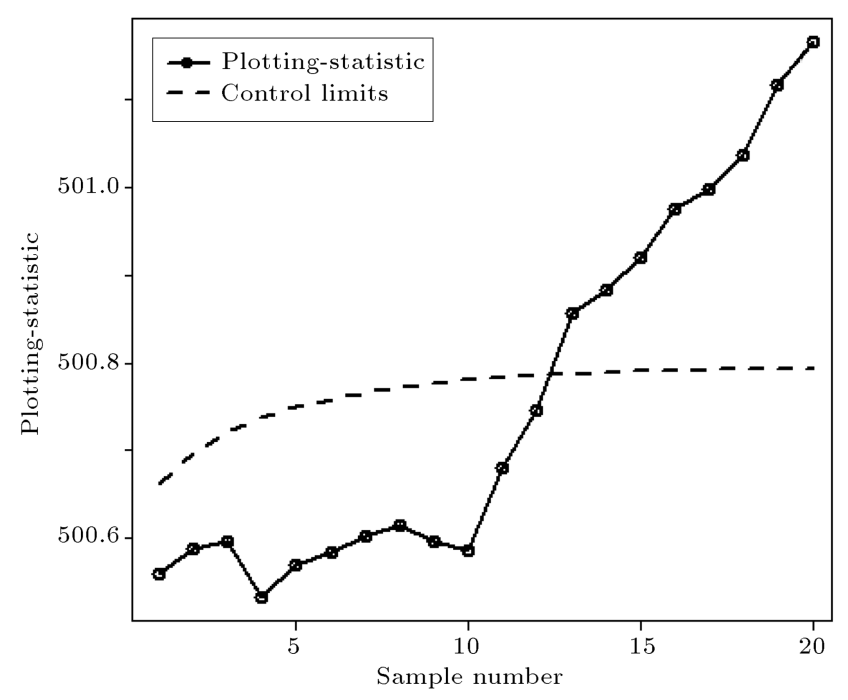

Figure 1. EWMA control chart with measurement error.

from \#13 to \#20. In Figure 3, for the upper-sided proposed control chart with measurement error, the out-of-control signals are triggered from \#11 to \#20, with $\lambda=0.1$ and $h=21.96$. According to Figures $1-3$, it is concluded that the proposed control chart provided better performance than CUSUM and EWMA control charts in the presence of measurement error.

\section{Conclusion and ercommendation}

This study reported the effect of measurement error on the performance of the mixed Exponentially Weighted

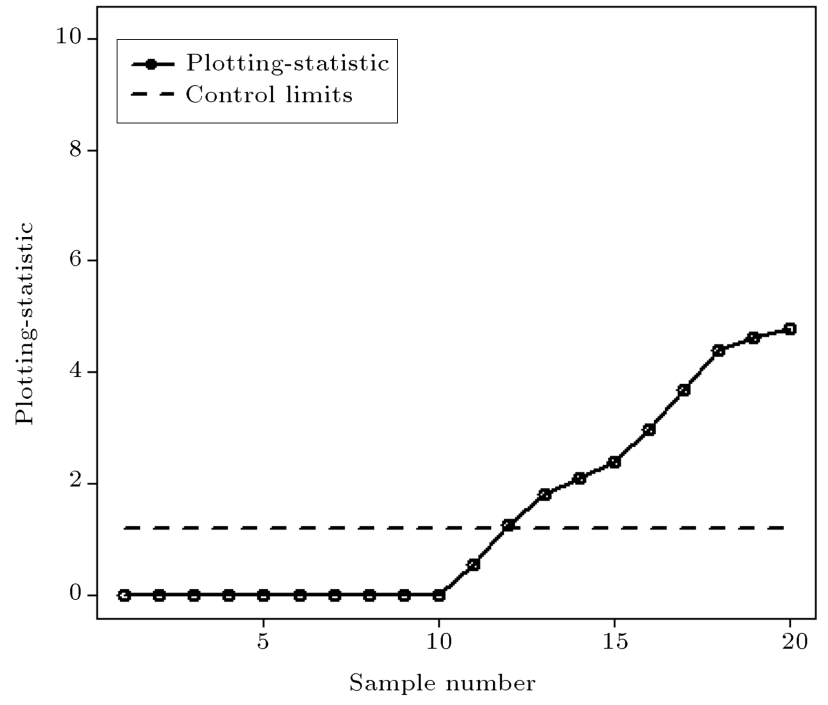

Figure 2. CUSUM control chart with measurement error.

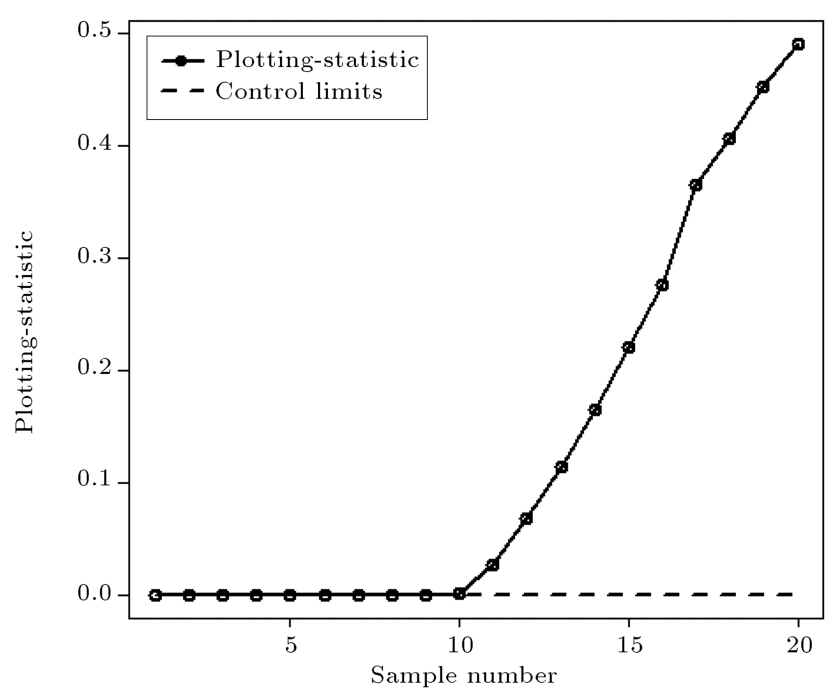

Figure 3. Mixed EWMA-CUSUM control chart with measurement error.

Moving Average-Cumulative Sum (EWMA-CUSUM) control chart. According to the results, the measurement error had a negative impact on the performance of the mixed EWMA-CUSUM control chart. The effect of measurement error was studied using covariates, multiple measurements, and linearly increasing variance methods with different sample sizes. It was concluded that the performance of the mixed EWMA-CUSUM control chart changed due to measurement error. The ARL (SDRL) increased, meaning that the measurement error had adverse effects on the performance of the mixed EWMA-CUSUM control chart. Further, it was observed that the multiple measurement method was a remedy against the adverse effect of measurement error. In most of real-life situations measurement error exists so it is recommended to discuss all the control charts with the measurement error. 


\section{Acknowledgments}

The author is thankful to Prof. Fu-Kwun Wang for his help regarding the $\mathrm{R}$-programming.

\section{References}

1. Roberts, S. "Control chart tests based on geometric moving averages", Technometrics, 1(3), pp. 239-250 (1959).

2. Page, E.S. "Continuous inspection schemes", Biometrika, 41(1/2), pp. 100-115 (1954).

3. Abbas, N., Riaz, M., and Does, R.J. "Mixed exponentially weighted moving average-cumulative sum charts for process monitoring", Quality and Reliability Engineering International, 29(3), pp. 345-356 (2013).

4. Abujiya, M.A.R., Riaz, M., and Lee, M.H. "Enhancing the performance of combined Shewhart-EWMA charts", Quality and Reliability Engineering International, 29(8), pp. 1093-1106 (2013).

5. Zaman, B., Riaz, M., Abbas, N., et al. "Mixed cumulative sum-exponentially weighted moving average control charts: an efficient way of monitoring process location", Quality and Reliability Engineering International, 31(8), pp. 1407-1421 (2015).

6. Ajadi, J.O., Riaz, M., and Al-Ghamdi, K. "On increasing the sensitivity of mixed EWMA-CUSUM control charts for location parameter", Journal of Applied Statistics, 43(7), pp. 1262-1278 (2016).

7. Riaz, A., Noor-ul-Amin, M., Shehzad, M.A., et al. "Auxiliary information based mixed EWMA-USUM mean control chart with measurement error", Iranian Journal of Science Technology, Transactions A: Science, 43(6), pp. 2937-2949 (2019).

8. Linna, K.W. and Woodall, W.H. "Effect of measurement error on Shewhart control charts", Journal of Quality Technology, 33(2), pp. 213-222 (2001).

9. Maravelakis, P., Panaretos, J., and Psarakis, S. "EWMA chart and measurement error", Journal of Applied Statistics, 31(4), pp. 445-455 (2004).

10. Mittag, H.-J. and Stemann, D. "Gauge imprecision effect on the performance of the XS control chart", Journal of Applied Statistics, 25(3), pp. 307-317 (1998).

11. Maravelakis, P.E. "Measurement error effect on the CUSUM control chart", Journal of Applied Statistics, 39(2), pp. 323-336 (2012).
12. Noor-ul-Amin, M., Riaz, A., and Safeer, A. "Exponentially weighted moving average control chart using auxiliary variable with measurement error", Communications in Statistics-Simulation and Computation (2019).

13. Ewan, W. and Kemp, K. "Sampling inspection of continuous processes with no autocorrelation between successive results", Biometrika, 47(3/4), pp. 363-380 (1960).

14. Montgomery, D.C., Introduction to Statistical Quality Control, John Wiley \& Sons (New York) (2009).

15. Bennett, C.A. "Effect of measurement error on chemical process control", Industrial Quality Control, 10(4), pp. 17-20 (1954).

16. Hawkins, D.M. and Olwell, D.H., Cumulative Sum Charts and Charting for Quality Improvement, Springer Science \& Business Media (2012).

17. Walden, C.T. "An analysis of variables control charts in the presence of measurement errors", Mississippi State University, Department of Industrial Engineering (1990).

18. Montgomery, D.C. and Runger, G.C. "Gauge capability and designed experiments. Part I: basic methods", Quality Engineering, 6(1), pp. 115-135 (1993).

19. Abbas, N. "Memory-type control charts in statistical process control", Universiteit van Amsterdam [Host] (2012).

20. Tran, K.P., Castagliola, P., and Balakrishnan, N. "On the performance of Shewhart median chart in the presence of measurement errors", Quality and Reliability Engineering International, 33(5), pp. 10191029 (2017).

\section{Biography}

Muhammad Noor-ul-Amin received his $\mathrm{PhD}$ degree from NCBA\&E, Lahore, Pakistan. He has working experience in teaching and research at various universities including the Virtual University of Pakistan, University of Sargodha, Pakistan, and the University of Burgundy, France. He is currently working as an Associate Professor at COMSATS University - Lahore Campus, Islamabad. His research interests include sampling techniques and control charting techniques. $\mathrm{He}$ is an HEC approved supervisor. 